\title{
Thermomagnetic Convection of Magnetic Fluid in an Annular Space under a Non-Uniform Magnetic and Thermal Field
}

\author{
Jun Liu \\ Tochigi R\&D Center, Keihin-Corp, Tochigi, Japan \\ Email: liujun3@hotmail.com
}

How to cite this paper: Liu, J. (2017) Thermomagnetic Convection of Magnetic Fluid in an Annular Space under a NonUniform Magnetic and Thermal Field. Applied Mathematics, 8, 655-662. https://doi.org/10.4236/am.2017.85052

Received: February 12, 2017

Accepted: May 20, 2017

Published: May 23, 2017

Copyright $\odot 2017$ by author and Scientific Research Publishing Inc. This work is licensed under the Creative Commons Attribution International License (CC BY 4.0).

http://creativecommons.org/licenses/by/4.0/

\section{(c) (i) Open Access}

\begin{abstract}
Two-dimensional thermomagnetic convection of magnetic fluid possessing internal spin and relaxation of magnetization with high thermal sensitivity is numerically analyzed under a non-uniform magnetic and thermal field, using a spectral difference scheme, magnetic fluid is assumed to be placed between concentric cylinders, an azimuthal magnetic field being produced by an electric current though the inner cylinder, which is an adiabatic wall, whereas a half of the outer cylinder is kept at a high constant temperature and the rest half is at a lower constant temperature. As a result, in case of $\nabla \mathrm{T} \times \nabla|\mathrm{H}| \neq 0$, thermomagnetic convection is found to be produced, the convection pattern depends on electric current distribution, depending on which multiple or single circulation is produced, thus, it shows that thermomagnetic convection pattern is controlled by changing relative direction of temperature gradient to that of a magnetic field.
\end{abstract}

\section{Keywords}

Magnetic Fluid, Thermomagnetic Convection

\section{Introduction}

A familiar example of thermal convection is buoyancy-induced convection, where a driving body force resides in a force of gravity, another example is established in a heated magnetic fluid subject to a magnetic body force, which depends on a thermodynamic constitutive equation. The enhancement of convective heat transfer in magnetic fluids could be applied in cooling current-carrying conductors, and transmissionlines, especially in gravity-free space. Characteristics of thermomagnetic convection are investigated by many researchers, e.g. Finlayson (1970) [1], Polevikov \& Fertman (1977) [2], Busse \& Riahi (1982) [3], Stiles \& 
Kagan (1990) [4], Zebib (1996) [5], Fruh (2005) [6], Yamaguchi (2009) [7] and Siddiqa (2013) [8].

In this paper, two-dimensional thermomagnetic convection of magnetic fluid possessing internal spin and relaxation of magnetization with high thermal sensitivity is numerically analyzed under a non-uniform magnetic and thermal field, using a spectral finite difference schemes, and the thermomagnetic convection pattern of magnetic fluid within an annulus is numerical investigated with spatial distributions of magnetic field variations.

\section{Analysis}

\subsection{Physical Model}

Analyzed is Two-dimensional thermomagnetic convection followed by a steadystate natural convection of magnetic fluid as shown in Figure 1, where a conductive wire of radius $R_{0}$ with a direct current $I_{0}$ is placed in the center of an annular space filled with a magnetic fluid of magnetic permeability $\mu_{0}$. It is assumed that magnetic fluid is magnetized by a magnetic field $H$ due to an external magnetic field from a wire current and an induction magnetic field of the magnetic fluid, thosemagnetic fluid is assumed to be at rest up to time $t=0$ with a uniform temperature $T_{b}$ and at $t>0$ a half part of the outside circumference is assumed to be fixed at a high temperature $\left(T_{h}\right)$, and the rest half part of the outside circumference at a low temperature $\left(T_{1}\right)$.

The following assumption applies:

1) The radius of wire is much smaller than that of the inside circle $\left(R_{0} \ll R_{1}\right)$.

2) The effect of magnetic field is limited within the distance $50 R_{2}$.

3) The magnetic permeability of magnetic fluid is equal to that of vacuum.

4) The magnetic fluid is incompressible fluid.

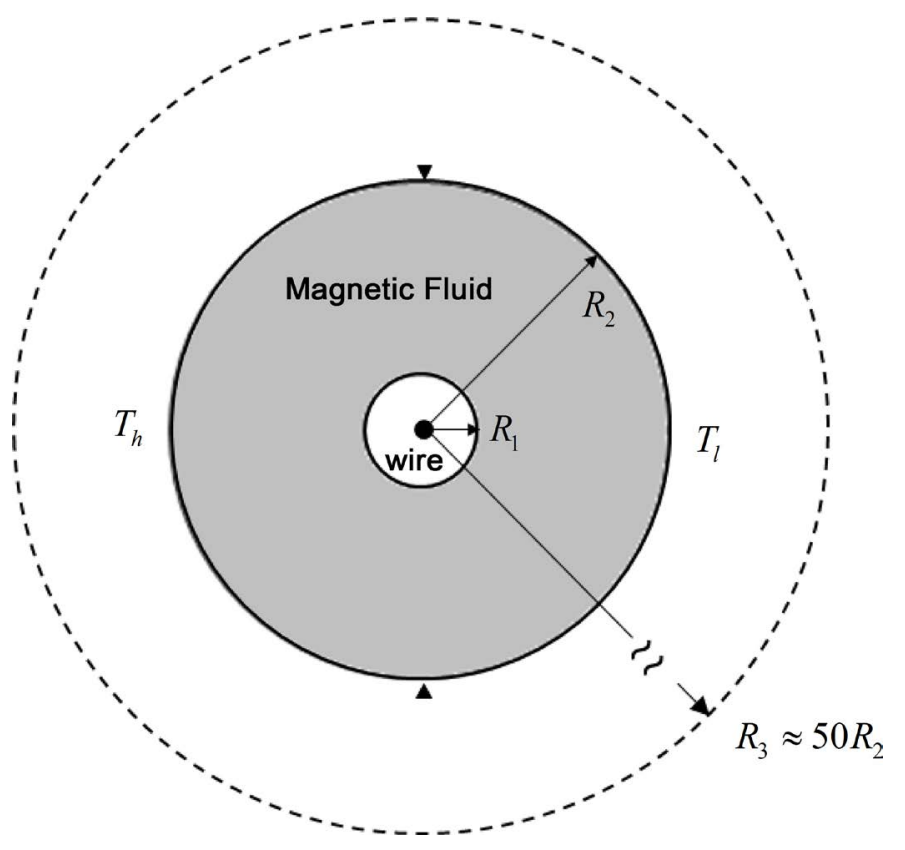

Figure 1. The analytical region. 


\subsection{Governing Equations of an Incompressible Magnetic Fluid}

The equation of continuity is

$$
\nabla \cdot v=0
$$

The momentum equation neglecting gravity force is

$$
\rho \frac{D \boldsymbol{v}}{D t}=-\nabla p+\eta \nabla^{2} \boldsymbol{v}+\nabla \cdot(\boldsymbol{B H})+\frac{I}{t_{s}} \nabla \times(\boldsymbol{\Omega}-\boldsymbol{\omega}),
$$

where $t$ is time, $\boldsymbol{v}$. velocity, $\rho$ : mass density of magnetic fluid, $p$ : pressure, $\eta$ : viscosity of magnetic fluid in absence of a magnetic field, $I$ : average inertia moments of particles per unit volume, $t_{s}$ : the relaxation time of internal spin rotation, $H$ : magnetic field, $B$ : magnetic induction, $\Omega$ : internal spin rate, $\omega$ : effective rate of rotation of a fluid element $\equiv(1 / 2) \nabla \times v$.

The internal angular momentum equation is

$$
I \frac{D \boldsymbol{\Omega}}{D t}=\gamma I \nabla^{2} \boldsymbol{\Omega}-\frac{I}{t_{s}}(\boldsymbol{\Omega}-\boldsymbol{\omega})+\mu_{0} \boldsymbol{M} \times \boldsymbol{H},
$$

where $\gamma$ is a dissipation coefficient of inertia spin moment, $M$ : magnetization of the magnetic fluid. The magnetization relaxation equation, Shliomis (1974) [9], is

$$
\frac{D \boldsymbol{M}}{D t}=\boldsymbol{\Omega} \times \boldsymbol{M}-\frac{1}{t_{b}}\left(\boldsymbol{M}-\boldsymbol{M}_{0}\right),
$$

where $t_{b}$ is a relaxation time of the particle rotation by Brownian rotation motion, and $M_{0}$ is the equilibrium magnetization of the magnetic fluid, and magnitude is given by

$$
\left|\boldsymbol{M}_{0}\right|(|\boldsymbol{H}|, T)=\phi M_{s}(T) \mathrm{L}\left(\frac{\pi \mu_{0} M_{s}(T) d^{3}|\boldsymbol{H}|}{6 k T}\right),
$$

where $\mathrm{L}(x)=\operatorname{coth} x-1 / x$ is a Langevin function, $T$ : temperature, $k$ : Boltzmann constant, $d$ : diameter of the particle, $\phi$ : volume fraction of ferromagnetic particles, $M_{s}$ : magnetic moment of a particle, if $T \leq 0.8 T_{c}\left(T_{c}\right.$ : temperature of Curie point),

$$
M_{s}(T) \approx M_{s}(0)\left(1-\frac{k T}{n M_{B} H_{M}}\right),
$$

where $M_{B}$ : Bohrmagneton, $n$ : number of spin perunitatom of ferromagntic metal, $H_{M}$ : molecular field.

The energy equation can be derived by Cowley and Rosensweig [10] as

$$
\rho C_{m} \frac{D T}{D t}-\mu_{0} \rho T K\left(\frac{D|\boldsymbol{H}|}{D t}\right)=k_{1} \nabla^{2} T+\Phi,
$$

where $C_{m}$ is the heat capacity at constant volume with a magnetic field, $K$ : pyromagnetic coefficient of magnetic fluid, $k_{1}$ : the thermal conductivity, $\Phi$ : a viscous dissipation term usually negligible. The Maxwell's equation for a non-conducting fluid with no displacement current becomes

$$
\nabla \times \boldsymbol{H}=0, \nabla \cdot \boldsymbol{B}=0,
$$




$$
\boldsymbol{B}=\mu_{0}(\boldsymbol{H}+\boldsymbol{M}) .
$$

\subsection{Coordinate System}

Cartesian coordinate $(x, y)$ is fixed in the center of an annular pipe, the $y$-axis being vertically upward, Relationship between cylindrical and Cartesian coordinates is

$$
x+i y=R \mathrm{e}^{i \theta},
$$

where, a spectral finite difference method is applied for solving Equation (1) Equation (8), and primary variables are expressed in a Fourier series to the circumferential $\theta$-direction.

\subsection{Equation of the Distribution of Magnetic Field}

Introduced are a magnetic induction stream functions, $\psi_{i}^{m}$ :

$$
\begin{gathered}
B_{i x}=\frac{\partial \psi_{i}^{m}}{\partial y}, B_{i y}=-\frac{\partial \psi_{i}^{m}}{\partial x}, \\
\psi_{i}^{m} \equiv \overline{\psi_{i}^{m}}-\frac{\mu_{0} I_{0}}{2 \pi} \ln R,
\end{gathered}
$$

where $i=1,2,3$ correspond to the following three regions:

- $R_{0} \leq R \leq R_{1}$ :

$$
\nabla^{2} \overline{\psi_{1}^{m}}=0
$$

- $R_{1} \leq R \leq R_{2}$ :

$$
\frac{1}{\mu_{0}} \nabla^{2} \overline{\psi_{2}^{m}}+\left(\frac{M_{\theta}}{R}+\frac{\partial M_{\theta}}{\partial R}-\frac{1}{R} \frac{\partial M_{R}}{\partial \theta}\right)=0,
$$

- $R_{2} \leq R \leq 50 R_{2}$ :

$$
\nabla^{2} \overline{\psi_{3}^{m}}=0 .
$$

where $M_{R}$ and $M_{\theta}$ are $R$ - and $\theta$-component of magnetization of the magnetic fluid, $\mathbf{M}$.

\subsection{Boundary Conditions}

The boundary conditions used are as follows:

- At $R=R_{1}$, no-slip, no-spin, and thermal insulation:

$$
[\boldsymbol{v}]_{R=R_{1}}=0, \quad[\boldsymbol{\Omega}]_{R=R_{1}}=0, \quad\left(\frac{\partial T}{\partial R}\right)_{R=R_{1}}=0,
$$

- At $R=R_{2}$, no-slip, no-spin, and Dirichlet temperature condition:

$$
\begin{gathered}
{[v]_{R=R_{2}}=0, \quad[\Omega]_{R=R_{2}}=0,} \\
T\left(\theta, R_{2}\right)=T_{h} \text { for } \theta \in\left(\frac{\pi}{2}, \frac{3}{2} \pi\right), \\
T\left(\theta, R_{2}\right)=T_{l} \text { for } \theta \in\left[0, \frac{\pi}{2}\right] \cup\left[\frac{3}{2} \pi, 2 \pi\right] .
\end{gathered}
$$


The normal component of the magnetic induction and the tangential component of the magnetic field are continuous across the interface between dissimilar regions,

$$
\begin{aligned}
& \text { at } R=R_{1}:\left[B_{1}\right]_{n}=\left[B_{2}\right]_{n},\left[H_{1}\right]_{t}=\left[H_{2}\right]_{t}, \\
& \text { at } R=R_{2}:\left[B_{2}\right]_{n}=\left[B_{3}\right]_{n},\left[H_{2}\right]_{t}=\left[H_{3}\right]_{t},
\end{aligned}
$$

where the subscript $n$ and $t$ denote normal and tangential component. Then the boundary condition of Equations 12(a)-(c) are given by

$$
\begin{gathered}
\overline{\varphi_{1}^{m}}=0, \quad \frac{\partial \overline{\varphi_{1}^{m}}}{\partial R}=0 \quad(R=0), \\
\frac{\partial \overline{\varphi_{1}^{m}}}{\partial R}=\frac{\partial \overline{\varphi_{2}^{m}}}{\partial R}+\mu_{0} M_{\theta} \quad\left(R=R_{1}\right), \\
\frac{\partial \overline{\varphi_{3}^{m}}}{\partial R}=\frac{\partial \overline{\varphi_{2}^{m}}}{\partial R}+\mu_{0} M_{\theta} \quad\left(R=R_{2}\right), \\
\overline{\varphi_{3}^{m}}=0 \quad\left(R=50 R_{2}\right) .
\end{gathered}
$$

\section{Results}

Assuming that the magnetic particle of magnetic fluid is iron oxide, and that the carrier liquid is hydrocarbon, the following constants and parameters of magnetic fluid are used

$$
\begin{aligned}
& C_{m}=2.09 \times 10^{3} \mathrm{~J} \cdot \mathrm{K}^{-1} \cdot \mathrm{kg}^{-1}, \quad d=1.0 \times 10^{-8} \mathrm{~m}, \quad H_{M}=1.7 \times 10^{9} \mathrm{~A} \cdot \mathrm{m}^{-1}, \\
& I=1.0 \times 10^{-4} \mathrm{~kg} \cdot \mathrm{m}^{-1}, \quad k=1.38 \times 10^{-23} \mathrm{~J} \cdot \mathrm{K}^{-1}, \quad k_{1}=0.22 \mathrm{~J} \cdot \mathrm{K}^{-1} \cdot \mathrm{m}^{-1} \cdot \mathrm{s}^{-1}, \\
& K=220 \mathrm{~A} \cdot \mathrm{m}^{-1} \cdot \mathrm{K}^{-1}, \quad M_{B}=1.17 \times 10^{-29} \mathrm{~A} \cdot \mathrm{m}^{-1}, M_{s}(0)=3.18 \times 10^{4} \mathrm{~A} \cdot \mathrm{m}^{-1}, \\
& n=2.2, \quad \phi=0.041, \eta=0.2 \mathrm{~Pa} \cdot \mathrm{s}, \quad t_{b}=7.6 \times 10^{-7} \mathrm{~s}, \quad t_{s}=4.17 \times 10^{-11} \mathrm{~s}, \\
& \mu_{0}=4 \pi \times 10^{-7} \mathrm{H} \cdot \mathrm{m}^{-1}, \quad \rho=1.22 \times 10^{3} \mathrm{~kg} \cdot \mathrm{m}^{-3} .
\end{aligned}
$$

\subsection{Magnetic Body Force of Magnetic Fluid}

Since the relaxation time $t_{\mathrm{s}}\left(\sim 10^{-11} \mathrm{~s}\right)$ of internal spin rotation and the dissipation coefficient $\gamma\left(\approx t_{b} / 2 \sim 10^{-7} \mathrm{~s}^{-1}\right)$ of inertia spin moment is very small, Equation (3) can be reduced as

$$
\frac{I}{t_{s}}(\boldsymbol{\Omega}-\boldsymbol{\omega})=\mu_{0} \boldsymbol{M} \times \boldsymbol{H},
$$

and substituting Equation (18) into Equation (2) leads to an approximate momentum equation:

$$
\rho \frac{D \boldsymbol{v}}{D t}=-\nabla p+\eta \nabla^{2} \boldsymbol{v}+\mu_{0}(\boldsymbol{M} \cdot \nabla) \boldsymbol{H}
$$

and then the curl of the magnetic body force is given by

$$
\nabla \times \mu_{0}(\boldsymbol{M} \cdot \nabla) \boldsymbol{H}=\mu_{0} \frac{\partial \chi}{\partial T}[\nabla T \times(\boldsymbol{H} \cdot \nabla) \boldsymbol{H}],
$$

where $\chi(=\chi(T,|H|))$ is the magnetic susceptibility of magnetic fluid, and the magnetization of the magnetic fluid can be written as $\boldsymbol{M}=\chi \boldsymbol{H}$. As for the Equation (20), the producing condition of thermomagnetic convection is derived in the gravity-free space as $\nabla T \times \nabla|\boldsymbol{H}| \neq 0$. 


\subsection{Numerical Results}

In the case of $I_{0}=10 \mathrm{~A}, T_{h}-T_{1}=20 \mathrm{~K}$ and $R_{1} / R_{2}=0.2$, the isotherms, streamlines and dimensionless magnetization field strengths with the position of electric current wire varies are displayed as shown in Figures 2-4, where all quantities are made dimensionless base on a length $L_{0}\left(=R_{2}\right)$, a temperature $T_{0}\left(=T_{h}-T_{1}\right)$, and reference velocity $V_{0}\left(=H_{0} \sqrt{\mu_{0} / \rho}\right), H_{0}\left(=\frac{I_{0}}{2 \pi L_{0}}\right)$ is representative magnetic field. It shows that the convection pattern depends on electric current distribution, depending on which multiple or single circulation is produced. Thus, it is found that thermomagnetic convection is controlled by changing relative direction of temperature gradient to that of a magnetic field.

\section{Conclusions}

1) For producing thermomagnetic convection of a magnetic fluid, a spatially non-uniform of temperature and a spatially non-uniform of external magnetic field are required so that $\nabla T \times \nabla|\boldsymbol{H}| \neq 0$.

(y)

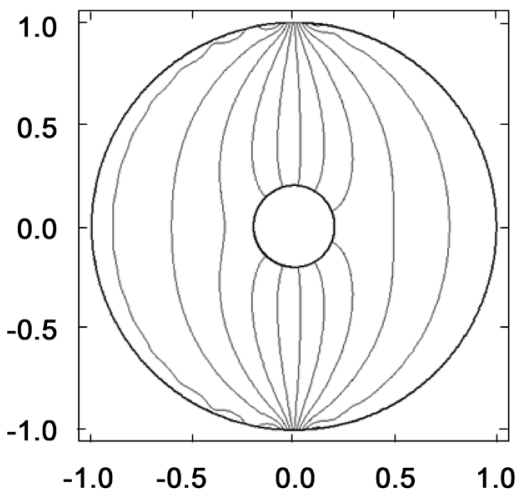

(y)

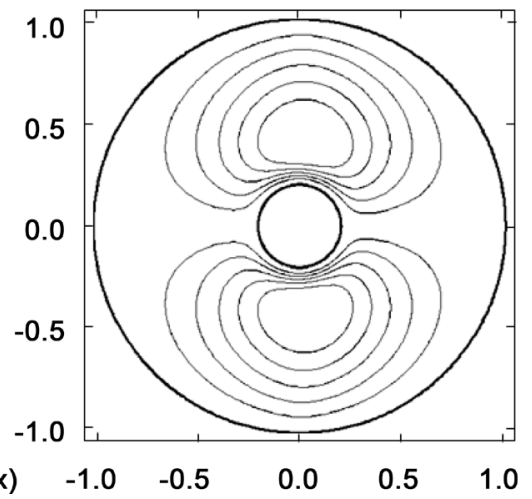

(y)

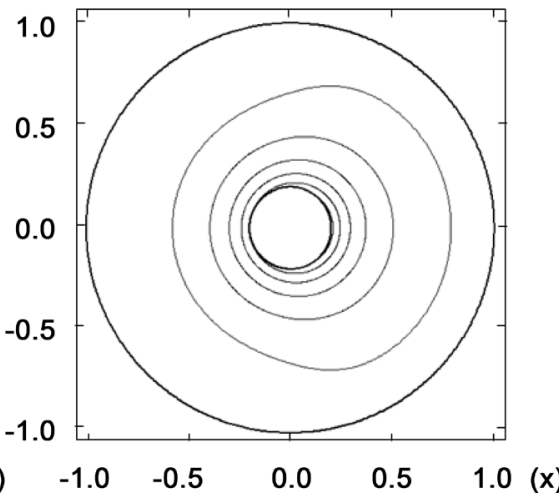

Figure 2. Isotherms (at an interval of 0.1), streamlines(at an interval of $1.0 \times 10^{-7}$ stream function) and dimensionless magnetization field strengths (at an interval of $1.0 \times 10^{-3}$ with from 0.001 to 0.007$)$ in the case of the electric current wire fixed at $(x, y)=(0$, 0 ), where the direction of velocity along the center is leftward.

(y)

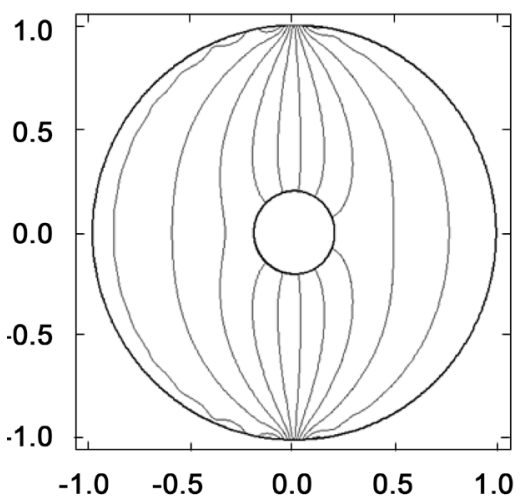

(y)

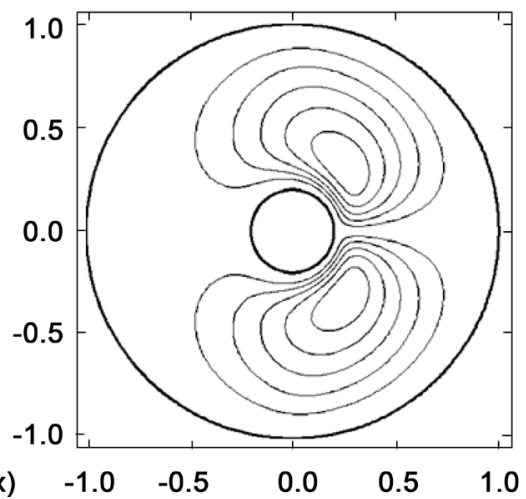

(y)

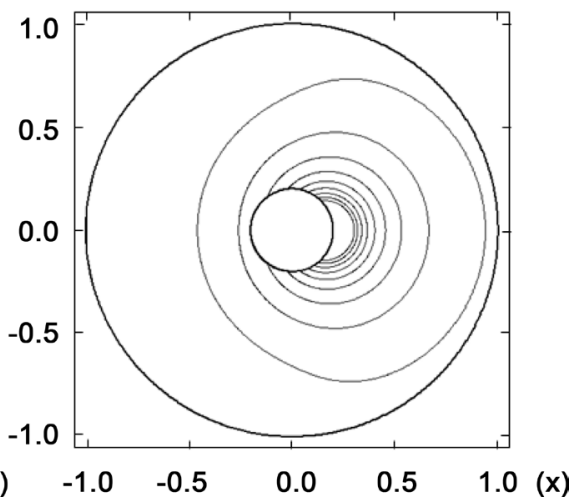

Figure 3. Isotherms (at an interval of 0.1), streamlines (at an interval of $2.0 \times 10^{-8}$ stream function) and dimensionless magnetization field strengths (at an interval of $2.0 \times 10^{-3}$ with from 0.002 to 0.02$)$ in the case of the electric current wire fixed at $(x, y)=(0.15$, 0 ), where the direction of velocity along the center is leftward. 
(y)

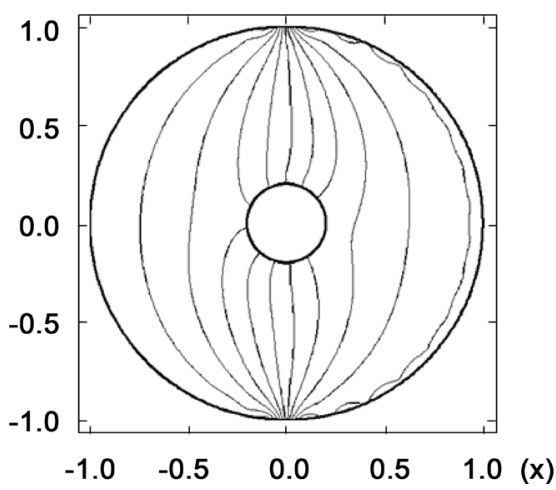

(y)

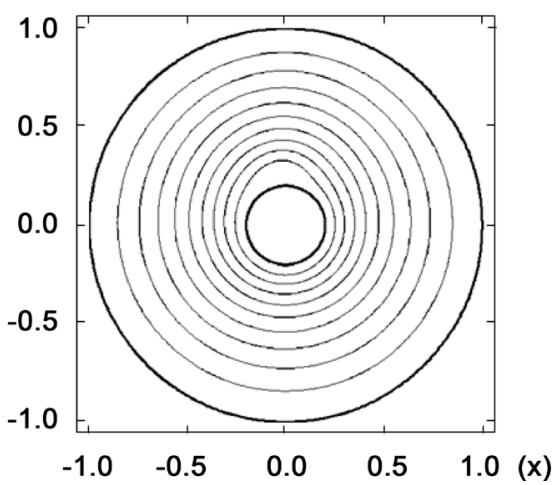

(y)

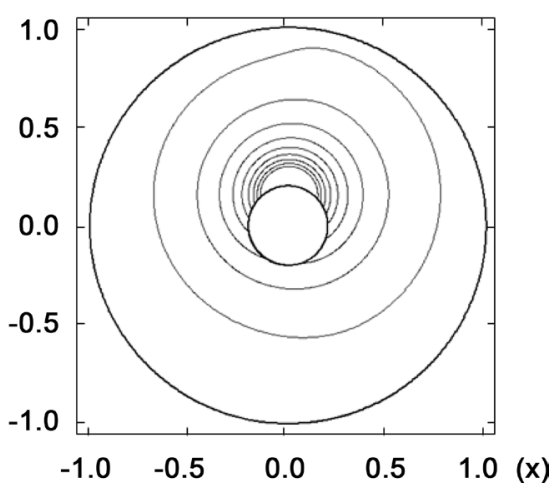

Figure 4. Isotherms (at an interval of 0.1 ), streamlines (at an interval of $1.0 \times 10^{-7}$ stream function) and dimensionless magnetization field strengths (at an interval of $2.0 \times 10^{-3}$ with from 0.002 to 0.02$)$ in the case of the electric current wire fixed at $(x, y)=(0$, $0.15)$, where the direction of flow is clockwise.

2) In the case that the electric current wire is fixed in center of annular pipe, two symmetrical circulating flows are produced.

3) If the position of electric current wire is different from the center, the center of the circulating flows moves to the side of lower or higher temperature depending on whether the location of electric current wire is to the lower or higher temperature side. Especially in the case of the position of electric current wire deviates greatly from the median line of temperature, only one circulating flow zone is produced.

\section{References}

[1] Finlayson, B.A. (1970) Convection Instability of Ferromagnetic Fluids. Journal of Fluid Mechanics, 40, 753-756. https://doi.org/10.1017/S0022112070000423

[2] Polevikov, K. and Fertman, E. (1977) Investigation of Heat Transfer through a Horizontal Layer of a Magnetic Liquid for the Cooling of Cylindrical Conductors with a Current. Magnetohydrodynamics, 13, 15-21.

[3] Busse, F.H. and Riahi, N. (1982) Patterns of Convection in Spherical Shells. Journal of Fluid Mechanics, 123, 283-301. https://doi.org/10.1017/S0022112082003061

[4] Stiles, P.J. and Kagan, M.J. (1990) Thermo-Convective Instability of a Horizontal Layer of Magnetic Fluid in a Strong Vertical Magnetic Field. Journal of Magnetism and Magnetic Materials, 85, 196-198. https://doi.org/10.1016/0304-8853(90)90050-Z

[5] Zebib, A. (1996) Thermal Convection in a Magnetic Fluids. Journal of Fluid Mechanics, 321, 121-136. https://doi.org/10.1017/S0022112096007665

[6] Fruh, W.J. (2005) Using Magnetic Fluids to Simulate Convection in a Central Force Field in the Laboratory. Nonlinear Processes in Geophysics, 12, 877-889.

[7] Yamaguchi, H., Niu, X.D., Zhang, X.R. and Yoshikawa, K. (2009) Experimental and Numerical Investigation of Natural Convection of Magnetic Fluids in a Cubic Cavity. Journal of Magnetism and Magnetic Materials, 321, 3665-3670. https://doi.org/10.1016/j.jmmm.2009.07.013

[8] Siddiqa, S., Hossain, M.A. and Saha, S.C. (2013) Natural Convection Flow in a Strong Cross Magnetic Field with Radiation. Journal of Fluids Engineering, 135, 051202-051209. https://doi.org/10.1115/1.4023854

[9] Shliomis, M.I. (1974) Magnetic Fluids. Soviet Physics Uspekhi (Engl. Trans), 17, 
$153-169$.

[10] Rosensweig, R.E. (1985) Ferrohydrodynamic. Cambridge University Press, Cambridge.

\section{Nomencalature}

$B$ : magnetic induction

$C_{m}$ : heat capacity at constant volume with a magnetic field

$d$ : diameter of the particle

$H$ : magnetic field

$H_{0}$ : external magnetic field

$H_{M}$ : molecular field

I: average inertia moments of particles per unit volume

$I_{0:}$ a direct current

$k$. Boltzmann constant

$k_{1}$ : thermal conductivity

$K$ : pyromagnetic coefficient of magnetic fluid

$M$ : magnetization of the magnetic fluid

$\boldsymbol{M}_{0}$ : equilibrium magnetization of the magnetic fluid

$M_{B}$ : Bohr magneton

$M_{s}$ : magnetic moment of a particle

$n$ : number of spin per unit atom of ferromagntic metal

$p$ : pressure

$R_{0}$ : radius of conductive wire

$R, \theta$. cylindrical coordinate system

t. time

T: temperature

$T_{\dot{c}}$ temperature of Curie point

$t_{b}$ : relaxation time of the particle rotation by Brownian rotation motion

$t_{s}$ : relaxation time of internal spin rotation

v. velocity

$x, y$. Cartesian coordinate system

$\gamma$ : dissipation coefficient of inertia spin moment

$\eta$ : viscosity of magnetic fluid in absence of a magnetic field

$\mu_{0}$ : permeability of the magnetic fluid

$\rho$ : mass density of magnetic fluid

$\psi:$ stream function

$\psi_{i}^{m}$ : a magnetic induction stream functions

$\phi$ : volume fraction of ferromagnetic particles

$\Phi$ : viscous dissipation term

$\chi$. magnetic susceptibility of magnetic fluid

$\omega$ : effective rate of rotation of a fluid element

$\Omega$ : internal spin rate 
Submit or recommend next manuscript to SCIRP and we will provide best service for you:

Accepting pre-submission inquiries through Email, Facebook, LinkedIn, Twitter, etc. A wide selection of journals (inclusive of 9 subjects, more than 200 journals)

Providing 24-hour high-quality service

User-friendly online submission system

Fair and swift peer-review system

Efficient typesetting and proofreading procedure

Display of the result of downloads and visits, as well as the number of cited articles Maximum dissemination of your research work

Submit your manuscript at: http://papersubmission.scirp.org/

Or contact am@scirp.org 